\title{
Application of sliding-window discretization and minimization of stochastic complexity for the analysis of fAFLP genotyping fingerprint patterns of Vibrionaceae
}

\author{
Peter Dawyndt, ${ }^{1}$ Fabiano L. Thompson, ${ }^{1}$ Brian Austin, ${ }^{2}$ Jean Swings, ${ }^{1}$ \\ Timo Koski ${ }^{3}$ and Mats Gyllenberg ${ }^{4} \dagger$ \\ ${ }^{1}$ Laboratorium voor Microbiologie, Universiteit Gent, B-9000 Gent, Belgium \\ ${ }^{2}$ School of Life Sciences, Heriot-Watt University, Edinburgh EH14 4AS, UK \\ ${ }^{3}$ Department of Mathematics, University of Linköping, S-58183 Linköping, Sweden \\ ${ }^{4}$ Department of Mathematics, University of Turku, FIN-20014 Turku, Finland
}

Correspondence

Peter Dawyndt

Peter.Dawyndt@ugent.be

\begin{abstract}
Minimization of stochastic complexity (SC) was used as a method for classification of genotypic fingerprints. The method was applied to fluorescent amplified fragment length polymorphism (fAFLP) fingerprint patterns of 507 Vibrionaceae representatives. As the current BinClass implementation of the optimization algorithm for classification only works on binary vectors, the original fingerprints were discretized in a preliminary step using the sliding-window band-matching method, in order to maximally preserve the information content of the original band patterns. The novel classification generated using the BinClass software package was subjected to an in-depth comparison with a hierarchical classification of the same dataset, in order to acknowledge the applicability of the new classification method as a more objective algorithm for the classification of genotyping fingerprint patterns. Recent DNA-DNA hybridization and 16S rRNA gene sequence experiments proved that the classification based on SC-minimization forms separate clusters that contain the fAFLP patterns for all representatives of the species Enterovibrio norvegicus, Vibrio fortis, Vibrio diazotrophicus or Vibrio campbellii, while previous hierarchical cluster analysis had suggested more heterogeneity within the fAFLP patterns by splitting the representatives of the above-mentioned species into multiple distant clusters. As a result, the new classification methodology has highlighted some previously unseen relationships within the biodiversity of the family Vibrionaceae.
\end{abstract}

\section{INTRODUCTION}

Bacterial taxonomy has a long tradition of using hierarchical cluster algorithms to establish taxonomies based on phenotypic and genotypic characteristics. Notwithstanding this, there has been concern about the subjective nature of the process (Priest \& Austin, 1993). It is appreciated that when using a hierarchical method of cluster analysis early

Published online ahead of print on 19 July 2004 as DOI 10.1099/ ijs.0.63136-0.

tPresent address: Department of Mathematics and Statistics, Rdf Nevanlinna Institute, University of Helsinki, FIN-00014, Finland.

Abbreviations: fAFLP, fluorescent amplified fragment length polymorphism; HMO, hypothetical median organism; SC, stochastic complexity.

An example of a stochastic-complexity-minimizing dendrogram for the fAFLP dataset of Vibrionaceae and the source code and a user manual for the BinClass software package are available as supplementary material in IJSEM Online. decisions in the construction process may preclude certain meaningful groupings at later stages (Sneath \& Sokal, 1973). Moreover, for a given dataset there might be many meaningful groupings that each reflect different aspects of the underlying relationships. Therefore, a single classification may give a distorted view of the multifaceted set of patterns. Consequently, if there are several meaningful groupings, a variety of cluster analysis techniques will be needed to reveal them all (Anderberg, 1973).

Inspired by these observations, the family of classification methods that optimize a given expression in information theory (such as entropy or stochastic complexity) was proven to be a complementary alternative to the hierarchical classifications (Gyllenberg et al., 1997b, 1998, 1999, 2002) used in bacterial taxonomy. However, the application of this kind of method for the classification of genotypic fingerprint patterns is hindered by the prerequisite that they only work on a vectorized data representation. Austin 
et al. (2004) demonstrated the impact that alternative discretization methods may have on the final classification result, and showed that sliding-window discretization results in a better conservation of the information content of the original fingerprint patterns, in comparison with other data discretization methods.

Thompson et al. (2001) analysed a set of 507 fluorescent amplified fragment length polymorphism (fAFLP) fingerprint patterns of Vibrionaceae strains based on a hierarchical classification using Ward's hierarchical cluster algorithm (Ward, 1963). This algorithm does not make a direct classification of the fingerprint patterns, but works on an intermediate similarity or dissimilarity matrix. In their report, Thompson et al. (2001) calculated this matrix using the Dice similarity coefficient $s_{\mathrm{D}}$ (Dice, 1945). From the hierarchical clustering a plain classification was derived by selecting a rather arbitrary $\alpha$-cut based on the intuition of the authors and the distribution of type and reference strains included in the dataset.

The current study has sought to reclassify the same set of fingerprint patterns based on more objective foundations and without taking into account prior knowledge about the bacterial strains. The classification method based on minimizing the stochastic complexity (SC) of a binary vector representation of the data (Gyllenberg et al., 1997a) was chosen as a representative method from the family of optimization algorithms for classification. This method has been implemented in the BinClass software package (Gyllenberg et al., 2001), which not only enables the calculation of an optimal classification in the sense of SC, but also allows the construction of an SC-driven hierarchy built on top of the optimal classification (Gyllenberg et al., 1998). An example of an SC-minimizing dendrogram for the fAFLP dataset of Vibrionaceae is available as supplementary material in IJSEM Online (Fig. S1).

The two classifications have been compared in depth, which has shown a global correlation between the major parts of the two groupings. However, differences between the classifications have stimulated the discovery of new relationships within the taxonomy of the Vibrionaceae strains, which have been confirmed by recent DNA-DNA hybridization and 16S rRNA gene sequence experiments (Thompson et al., $2003 a, b, c, d)$. These results prove the value of the new methodology as an alternative classification strategy in its own right, and the need to inspect a given dataset from different angles using different mathematical models in order to get a complete picture of all the relationships present.

\section{METHODS}

Bacterial strains. In this study we have analysed a total of 507 fAFLP (Janssen et al., 1996) fingerprint patterns from isolates of the family Vibrionaceae. The dataset was identical to that studied by Thompson et al. (2001), where the number of bands of the fAFLP profiles ranged from 46 to 164 , with a global mean of 107 bands and a standard deviation of 23. More detailed band statistics for each class resulting from the classification generated in the framework of this study are included in Table 1 . In their original report, Thompson et al. (2001) classified the fAFLP fingerprint patterns using Ward's hierarchical clustering algorithm (Ward, 1963). For pairwise fragment comparison, the Dice similarity coefficient $s_{\mathrm{D}}$ (Dice, 1945) was applied with a band position tolerance of $0.5 \%$ to compensate for misalignment of homologous bands due to technical imperfections. These calculations were performed using the BioNumerics software package (Applied Maths). After cluster delineation based on the results from previous studies and prior knowledge about the distribution of the type strains within the dataset, this classification strategy resulted in a set of 69 clusters (labelled A1 to A69) and four singleton fingerprint patterns (labelled U1 to U4).

Discretization of fAFLP fingerprint patterns. A classification strategy based on the optimization of an expression in information theory such as SC, which is a quantitative criterion for evaluation of the global goodness of a given classification with respect to the given dataset, requires that the fAFLP fingerprint patterns of the Vibrio isolates are transformed into a binary vector representation. Austin et al. (2004) have shown that the choice of discretization method used may have a major impact on the final classification. They proved that, among other algorithms, sliding-window discretization resulted in the binary vector representation with the highest conservation of the original information content of the fAFLP fingerprint patterns included in this study.

The sliding-window procedure is an extension of the well-known equal-width discretization method, controlled by two parameters: (i) the position tolerance parameter $\varepsilon$, which defines a window through which bands that are measured at different physical locations are logically considered as similar regardless of their position within the same fingerprint profile or within different profiles, thus compensating for misalignment of homologous bands due to technical imperfections; and (ii) the resolution of the method $\delta$, which controls the step size by which the window shifts over the continuous interval that must be sampled in a discrete manner. If the resolution of the method is smaller than the position tolerance, the different sampling windows used by the sliding-window method will overlap, in contrast to the equal-width method, where sampling windows never overlap with each other. This implies a fuzzyfication of the window boundaries in the resulting vector representation.

For the classification within the current study, the sliding-window discretization method was applied with $\varepsilon$ set to 0.007 and $\delta$ set to $0 \cdot 001$, in order to maximize the Pearson product-moment correlation $r$ with the $s_{\mathrm{D}}$ similarity matrix from the study of Thompson et al. (2001). After all, to enable an objective comparison of the different classification strategies applied to the same Vibrio/fAFLP dataset, it is important to mimic the same band position tolerance behaviour and reduce the effect of fragment comparison of the fingerprint patterns within the classification process. As a result, the fAFLP fingerprint patterns were transformed by the sliding-window discretization method into binary vectors of length 994 .

Classification of binary vectors. The binary vectors resulting from the sliding-window discretization procedure were classified using minimization of SC (Gyllenberg et al., 1997a), as it was implemented in the BinClass software package (Gyllenberg et al., 2001). This algorithm is an example of an unsupervised non-hierarchical classification method, insofar as it does not make use of prior knowledge or assumptions on the dataset other than the binary vector representation of its characteristics and its final result is a plain partitioning of the dataset into non-overlapping classes. The default parameter settings were chosen, except for the $-\mathrm{F}$ parameter (safety value), which was set to 50 , and the $-S$ parameter, which was set to 20. This has resulted in the classification with 64 classes (labelled BC1 to BC64) that is summarized in Table 1. The optimal SC found for the dataset was $739 \cdot 92$. The BinClass software package 
Table 1. BinClass classification based on data discretized by the sliding-window discretization method with the position tolerance parameter $\varepsilon$ set to 0.007 and the resolution of the method $\delta$ set to 0.001 (so that vector length $d=994$ )

BinClass run performed with command line settings (-F50 -S20), resulting in a classification with 64 classes and an SC of 739.92280. 1, Class identifier. 2, Size of class (number of strains $n$ ). 3, Mean number of bands (standard deviation) over all profiles in the class. 4, Minimal and maximal number of bands of all profiles in the class. 5, fAFLP cluster in classification by Thompson et al. (2001). 6, Name of fAFLP cluster as given by Thompson et al. (2001). 7, Frequency of original fAFLP cluster within class. 8, Mean Shannon code length of the class. 9, Class distortion. 10, Nearest class. 11, Hamming distance to nearest class. 12, Farthest class. 13, Hamming distance to farthest class. 14, Hamming distance between type strain and hypothetical median organism. 15, Shannon code length of type strain.

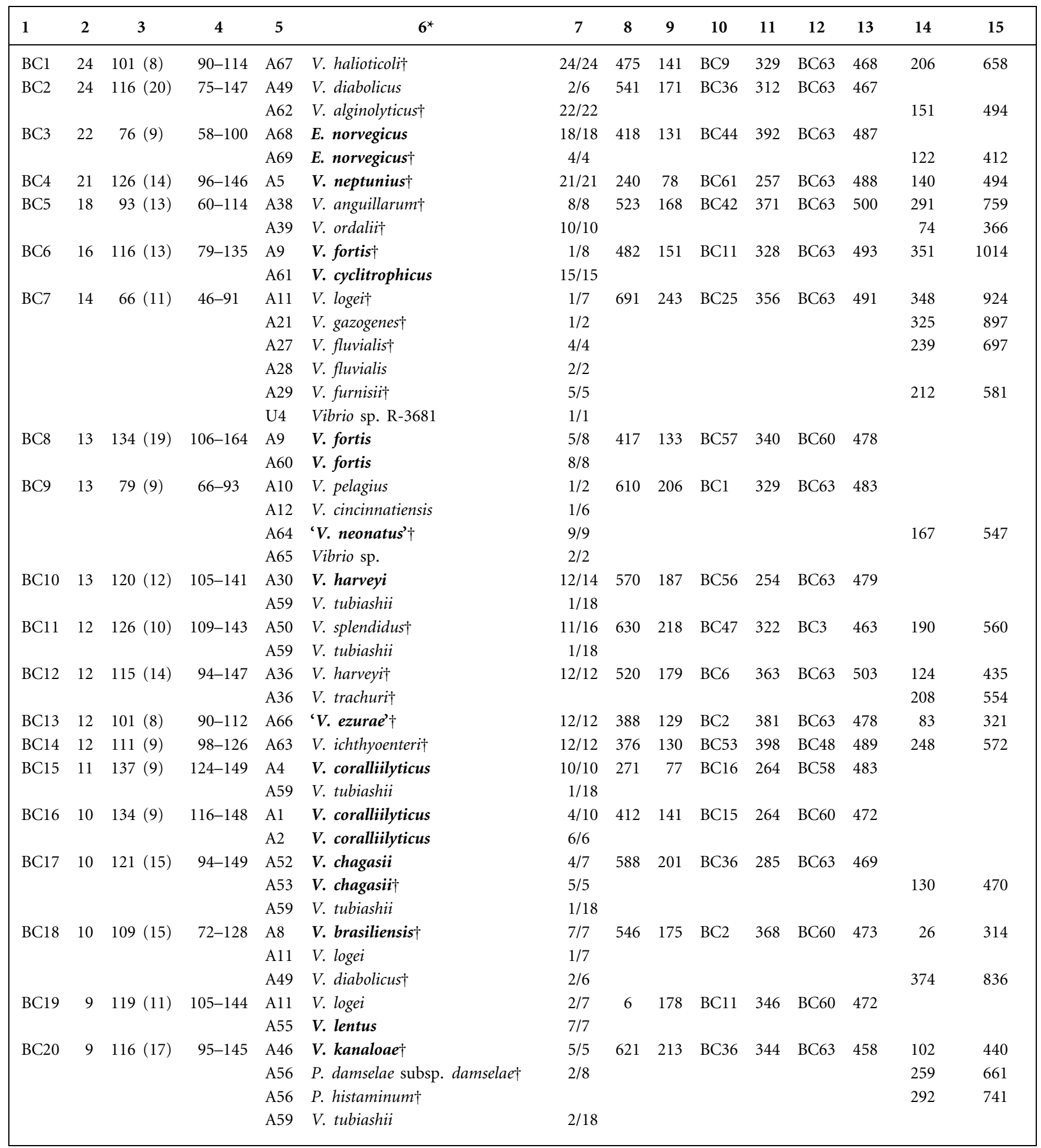


Table 1. cont.

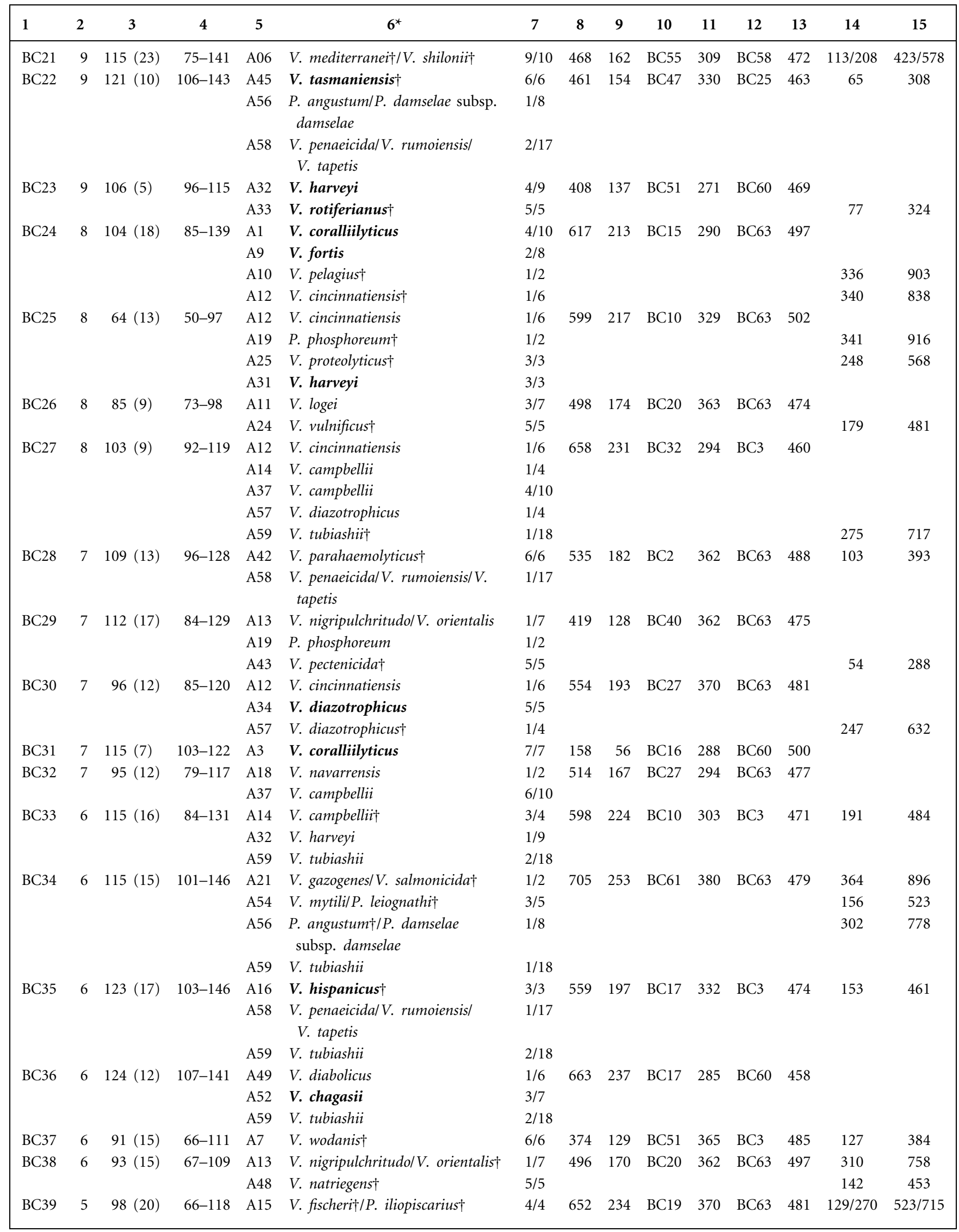


Table 1. cont.

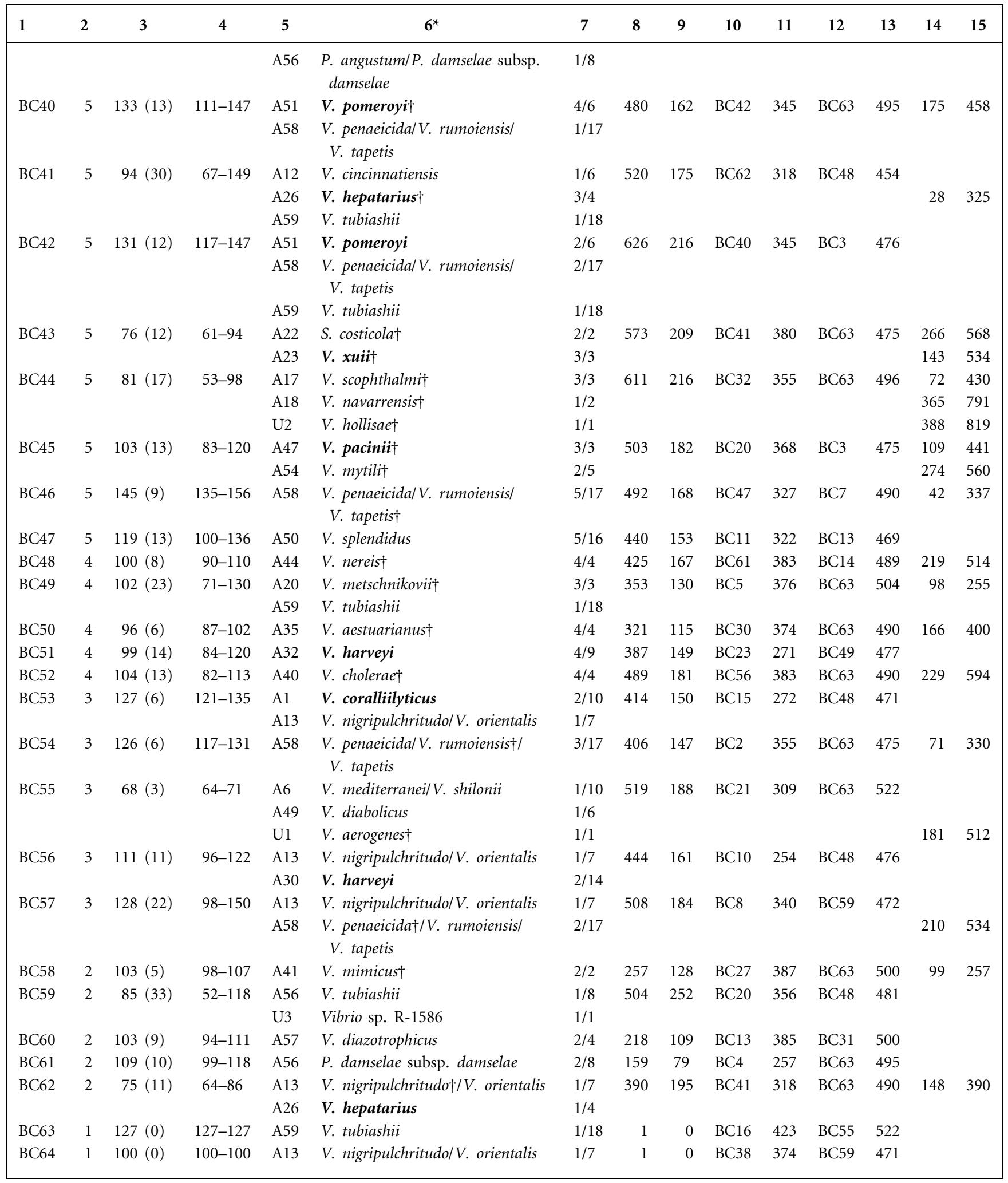

${ }^{\star}$ E., Enterovibrio; P., Photobacterium; S., Salinivibrio; V., Vibrio. Bold type indicates revised name since publication of the report by Thompson et al. (2001).

$\dagger$ Type strain position. 
automatically accommodated for monomorphic bands by discarding vector indexes that had the same binary value before performing the classification, hence taking only into account the bands that were polymorphic within the dataset.

The Hamming distance between two binary vectors is defined as the number of bits that are different, from which the distance between two classes can be defined as the mean pairwise Hamming distance between members of the two classes (Gyllenberg et al., 1999). The centroid of a class is by definition the vector giving the frequencies of 1's for the different attributes. By rounding off each component of the centroid to the nearest binary value ( 0 or 1$)$ one obtains the hypothetical median organism (HMO; Liston et al., 1963). The distortion of a class, defined as the mean number of bits by which the members of the class differ from the HMO (mean Hamming distance), can be regarded as a measure of the heterogeneity of a class. Shannon code length (Cover \& Thomas, 1991) between a class member and the centroid of the class was used as an alternative distance function, where the mean Shannon code length of all class members with respect to the centroid of the class gives an alternative quantifier for describing the heterogeneity of the class. For both the distortion and the mean Shannon code length it holds that classes with lower values for these parameters are more homogeneous than classes with higher values.

From a taxonomic viewpoint the fingerprint profiles of the type strains that were included in the dataset are fairly neatly distributed over the different classes that result from the BinClass classification (see Table 1), although this was not forced by any subjective decision-making within the classification strategy and the type strain information was not regarded as prior knowledge by the classification scheme. This proves that the partitioning of the fAFLP patterns from the current study by minimization of the SC generated classes that generally correspond well with the species delineated within the family Vibrionaceae. In this context, both the centroid and the HMO can be regarded as estimations of fictitious representatives for each class and thus, by extension, for the species that are represented by these classes. The Hamming distance to the HMO and the Shannon code length may then be employed as measures for evaluating the typicality of a pattern for the class it belongs to or, stated differently, they measure how typical a strain is of the species to which it is identified by a given classification procedure applied on a chosen set of characters of the strain. Since a type strain has to be designated when a species is first described and named, the nomenclatural type strain is nothing more than the name bearer of the species and is usually the first strain known (Buchanan, 1925). Hence, at the time of type strain selection so little information has yet been found out about the constellation of the species which will be represented by that strain that there is not enough statistical evidence in order to ensure that the type strain is indeed a typical strain (Sneath, 1984). Moreover, some of the type strains might be comparatively old and have lost useful characters due to gene loss caused by the long preservation time of the strains.

The source code and a user manual of the BinClass software package are available as supplementary materials in IJSEM Online. Due to the command line interface and the ANSI C compliance of the source code, the software easily compiles on most operating systems (e.g. Win32, UNIX and Linux). For the convenience of the readership, all BinClass-formatted input files and all the output files generated by the software package in the framework of this study are also included as supplementary materials, together with an executable version of the program that has been compiled to run on all Win32 platforms. One of the output files generated by the BinClass software package contains a complete description of the classification results, in which each of the 507 Vibrionaceae strains is accounted for. This information is far too extensive to be reported in detail within this publication.
Comparison of different classifications. In order to rate the value of minimizing SC for the classification of bacterial genotyping fingerprint patterns, the BinClass classification of the Vibrio/AFLP dataset was compared with the classification of the same dataset as described by Thompson et al. (2001). Fig. 1 shows a graphical representation of the comparison between these two classifications. In this representation, each row represents a class from the classification described previously by Thompson et al. (2001), with the assigned class identifier in the first column and the number of strains in the last column. Each column represents a class from the BinClass classification, with the assigned class identifier in the first row and the number of strains in the last row. The values in the row/column intersections represent the number of strains that the two corresponding classes have in common. The following section gives a detailed discussion about the similarities and differences found between the two classifications, together with the taxonomic implications for the Vibrio dataset.

\section{RESULTS AND DISCUSSION}

The 507 strains examined in this study formed 64 classes (BC1 to BC64), several of which (i.e. BC1, BC4, BC12, $\mathrm{BC} 13, \mathrm{BC} 14, \mathrm{BC} 31, \mathrm{BC} 37, \mathrm{BC} 48, \mathrm{BC} 50, \mathrm{BC} 52$ and $\mathrm{BC} 58$ ) corresponded exactly with classes obtained from the clustering of the same dataset using $s_{\mathrm{D}} / \mathrm{Ward}$ and an arbitrary cluster cut off value of $45 \%$ (Thompson et al., 2001). In addition, new relationships among former fAFLP clusters have been disclosed, many of which are in agreement with recent DNA-DNA hybridization and 16S rRNA gene sequence experiments.

Class BC1 contained 24 Vibrio halioticoli strains, with low pattern distortion (heterogeneity), and was most closely related to BC9, which constituted a new Vibrio species, 'Vibrio neonatus', phylogenetically related to $V$. halioticoli (Sawabe et al., 2002, 2004). Class BC2 had 24 strains, including the type strain of Vibrio alginolyticus and two Vibrio diabolicus strains, according to the clustering obtained by Thompson et al. (2001). Interestingly, these two $V$. diabolicus strains were originally identified by Vandenberghe et al. (1999), using phenotypic and genotypic techniques, as $V$. alginolyticus. The nearest class of BC2 was BC36, which included one $V$. diabolicus strain.

Class BC3 contained 22 Enterovibrio norvegicus strains. The combination of the two fAFLP clusters A68 and A69 in BC3 is in complete agreement with more recent analyses based on DNA hybridization and 16S rRNA gene sequences which proved that these two clusters are in fact a single species, E. norvegicus (Thompson et al., 2002). The nearest class to BC3 was BC44, which contains Vibrio hollisae. This is also the closest phylogenetic neighbour of Enterovibrio based on 16S rRNA gene analysis, having about $95 \%$ sequence similarity (Thompson et al., 2002). Class BC4 consisted of 21 Vibrio neptunius strains of remarkably low pattern diversity, while class BC5 merged all Vibrio anguillarum and Vibrio ordalii strains analysed. V. ordalii was described by Schiewe et al. (1981) to encompass biotype II of $V$. anguillarum. It is well known that these species are highly related, having nearly $100 \% 16 \mathrm{~S}$ rRNA gene similarity and $70 \%$ DNA-DNA similarity. Class BC6 contained the type 


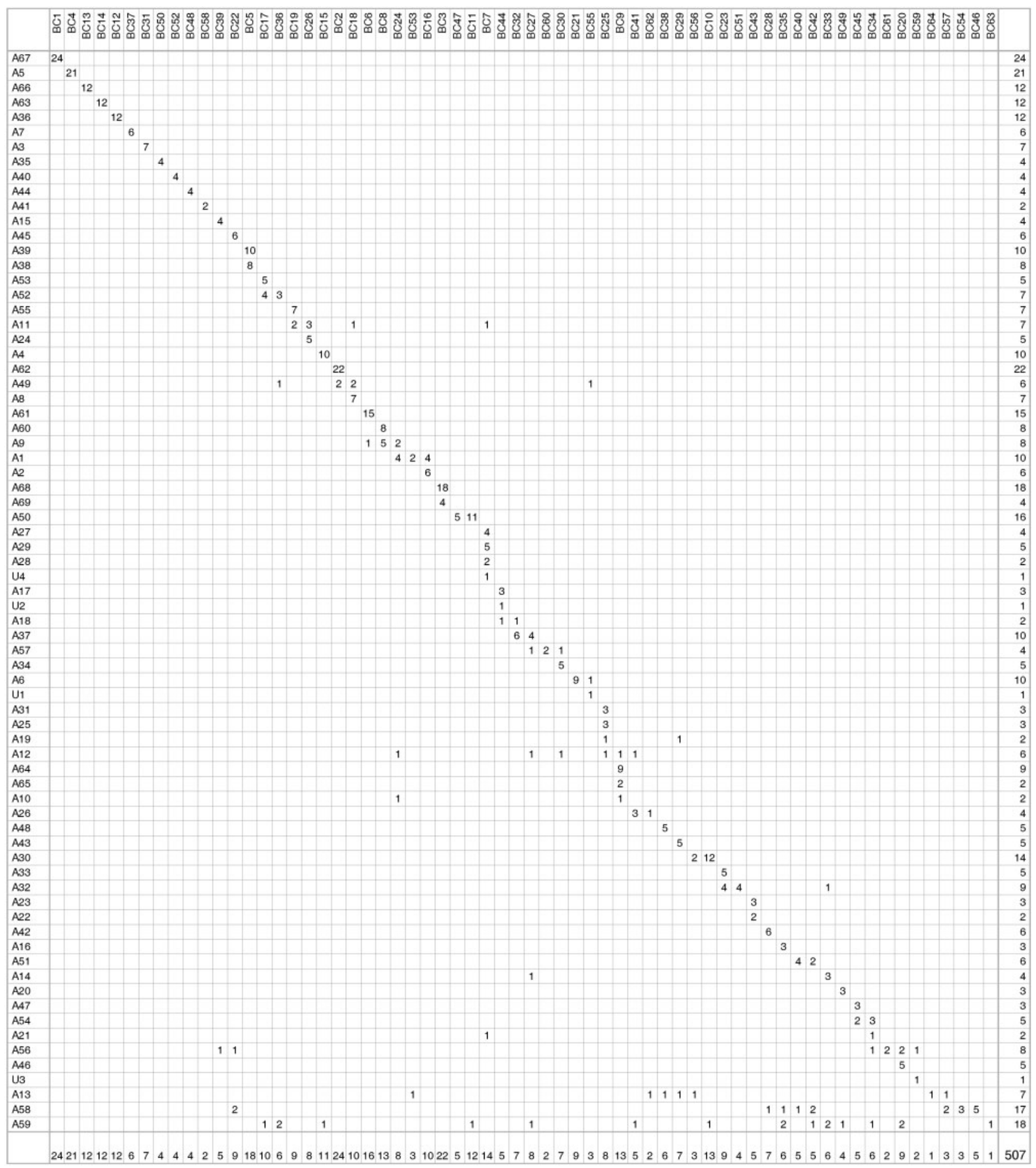

Fig. 1. Comparison of the classification described by Thompson et al. (2001) and the BinClass classification based on data discretized by the sliding-window method with the position tolerance parameter $\varepsilon$ set to 0.007 and the resolution of the method $\delta$ set to 0.001 (so that the vector length $d=994$ ). BinClass was run with command line settings $(-\mathrm{F} 50-\mathrm{S} 20$ ), resulting in a classification with 64 classes and an SC of 739.92280. Each row represents a class from the classification described previously by Thompson et al. (2001), with the assigned class identifier in the first column and the number of strains in the last column. Each column represents a class from the BinClass classification, with the assigned class identifier in the first row and the number of strains in the last row. The values in the row/column intersections represent the number of strains that the two corresponding classes have in common. 
strain of Vibrio fortis and 15 Vibrio cyclitrophicus strains, while most of the other members of $V$. fortis appeared in $\mathrm{BC} 8$ and $\mathrm{BC} 24$. $V$. fortis was proposed to encompass strains of the former fAFLP clusters A9 and A60 (Thompson et al., 2003a). The fact that the type strain of this species clustered apart from all other species members may suggest that the type strain of $V$. fortis is a species on its own and that this heterogeneous species may be split into new species in the future. Class BC8 merged five strains of the former fAFLP group A9 and all eight strains of A60, which correspond to the newly described $V$. fortis. Clearly BC8 differs from the type strain of $V$. fortis (in BC6).

Class BC7 consisted of 14 strains, including the type strains of Vibrio gazogenes, Vibrio fluvialis, Vibrio furnissii and Vibrio logei. A remarkable feature of this diverse class is the large distance from all the type strains to the HMO (Liston et al., 1963). It is reasonable that V. fluvialis and $V$. furnissii (former biotype of $V$. fluvialis) group together, although one would not expect the inclusion of the psychrophilic $V$. logei in this class. A $16 \mathrm{~S}$ rRNA genebased phylogenetic analysis of $V$. logei revealed that this organism is more related to the psychrophilic vibrios (e.g. Vibrio fischeri, Vibrio salmonicida, Vibrio wodanis) than to any of the species within BC7. Class BC9 $(n=13)$ contained nine strains of a new Vibrio species, Vibrio neonatus (Sawabe et al., 2004). This class included two other vibrios named Vibrio pelagius and Vibrio cincinnatiensis, which are most probably representatives of this new species. Classes $\mathrm{BC} 10, \mathrm{BC} 12, \mathrm{BC} 23, \mathrm{BC} 25, \mathrm{BC} 33, \mathrm{BC} 51$ and $\mathrm{BC} 56$ contained Vibrio harveyi strains, with the type strain of $V$. harveyi being in $\mathrm{BC} 12$, suggesting that this is a very diverse species. In contrast, $\mathrm{BC} 11(n=12)$ included the type strain and most reference strains of Vibrio splendidus and one strain of Vibrio tubiashii. This class was related to BC47, which contained all the remaining $V$. splendidus strains; these may be a variant of $V$. splendidus or a new species. Two strains of BC47 were found in the so-called ribotype cluster C described by Mácian et al. (2000). Strains originally allocated to $V$. tubiashii (Thompson et al., 2001) were repartitioned into different $\mathrm{BC}$ classes $(\mathrm{BC} 10, \mathrm{BC} 11, \mathrm{BC} 15$, $\mathrm{BC} 17, \mathrm{BC} 20, \mathrm{BC} 27, \mathrm{BC} 33, \mathrm{BC} 34, \mathrm{BC} 35, \mathrm{BC} 36, \mathrm{BC} 41, \mathrm{BC} 42$, $\mathrm{BC} 49, \mathrm{BC} 59$ and $\mathrm{BC} 63)$, with the type strain allocated to $\mathrm{BC} 27$. This suggests that the original $V$. tubiashii group as delineated by Thompson et al. (2001) was quite artificial.

Class BC13 contained a new Vibrio species, 'Vibrio ezurae' (Sawabe et al., 2002, 2004), while BC14 contained 12 Vibrio ichthyoenteri strains. Classes $\mathrm{BC} 15$ and $\mathrm{BC} 16$ included most Vibrio coralliilyticus strains (Ben-Haim et al., 2002), although BC24, BC31 and BC53 included four, seven and two strains of this species, respectively. It is quite remarkable that BC31 made a (homogeneous) cluster on its own, suggesting it is a variant of $V$. coralliilyticus. Strains of this class appear to be specialized in causing disease in Nodipecten nodosus bivalve larvae, while other $V$. coralliilyticus are known coral pathogens (Ben-Haim et al., 2002). Class BC17 consisted of 10 strains including the type strain of Vibrio chagasii and most of the reference strains of this new species (Thompson et al., 2003c). The nearest neighbour of $\mathrm{BC} 17$ was $\mathrm{BC} 36$, which contained three $V$. chagasii strains (LMG 13220, LMG 13222 and LMG 13239), suggesting that they may be yet another new species. In fact, a closer examination of the fAFLP patterns of $V$. chagasii strains and the DNA-DNA hybridization data indicates a large diversity within this species in support of the new grouping obtained here. In class BC18 two species, Vibrio brasiliensis and $V$. diabolicus, were merged. This is not an ideal situation, but the grouping might be just a reflection of the phylogenetic relatedness between the two species as they share approximately $98 \% 16 \mathrm{~S}$ rRNA gene similarity. BC19 included all Vibrio lentus strains and two V. logei strains. Interestingly, the nearest neighbour class to BC19 was BC11. It is well known that $V$. lentus and $V$. splendidus are highly related species. The strains allocated to $V$. logei in the previous fAFLP analysis (Thompson et al., 2001) were quite heterogeneous, as can be seen from the new partitioning of the strains. The type strain of $V$. logei appeared in $\mathrm{BC}$, but another one, two and three strains appeared in $\mathrm{BC} 18, \mathrm{BC} 19$ and $\mathrm{BC} 26$, respectively. These strains were supposed to be $V$. logei, however the results presented here undermine this assumption.

BC20 merged together three type strains (i.e. P. histaminum, Photobacterium damselae and Vibrio kanaloae), which might undermine the value of the new classification. However, $P$. damselae was originally clustered with Photobacterium angustum (Thompson et al., 2001). P. angustum appears now in BC34 along with the type strains of $V$. salmonicida and Photobacterium leiognathi. BC21 consisted of nine Vibrio mediterranei strains including the former type strain of Vibrio shilonii (Thompson et al., 2001). BC22 comprised six strains of a newly described species, Vibrio tasmaniensis (Thompson et al., 2003b), and three strains from two very heterogeneous fAFLP clusters, A56 and A58. Thompson et al. (2001) highlighted that the precise taxonomic allocation of isolates clustering with more than one type strain was unclear, requiring further investigation. Here we demonstrate the partitioning of such isolates by using minimization of SC, indicating the usefulness of this new approach.

BC23 contained Vibrio rotiferianus (Gomez-Gil et al., 2003) and four $V$. harveyi strains. According to Gomez-Gil et al. (2003) both species are highly related, and the results presented here may suggest that those four strains identified as $V$. harveyi are in fact $V$. rotiferianus. BC23 was related to BC51, which comprised four diverse $V$. harveyi isolates. Class BC24 included the type strains of $V$. cincinnatiensis and V. pelagius, whereas BC25 included those of Photobacterium phosphoreum and Vibrio proteolyticus. All these species were clearly separated in the clustering of Thompson et al. (2001), but with the SC-minimizing classification one may expect that certain species be grouped together. This fact may be just a reflection of the limitation of band patterns, which happen to give similar fingerprints between 
completely unrelated species (e.g. P. phosphoreum and $V$. proteolyticus). We inspected the original patterns of the species within BC25 and found large gaps, which will turn out as zeros in the binarized patterns used for comparisons.

Class BC26 consisted of five Vibrio vulnificus strains. This class also included three so-called $V$. logei strains. Two of these strains, i.e. VIB 523 and STD3-996, are clearly $V$. vulnificus representatives misidentified by Thompson et al. (2001). Arias et al. (1997) identified VIB 523 to the species $V$. vulnificus using phenotypic and genotypic techniques, while our 16S rRNA gene sequence of STD3-996 revealed $100 \%$ similarity to $V$. vulnificus. BC27 consisted of eight strains, including the type strain of $V$. tubiashii, while BC28 had seven strains, including the type strain of Vibrio parahaemolyticus. BC28 was closely related to BC2, which contained $V$. alginolyticus (a former variant of $V$. parahaemolyticus). BC29 comprised the species Vibrio pectenicida and two other strains which were probably misidentified in the former analysis by Thompson et al. (2001). Surprisingly, class BC30 put together the former fAFLP cluster A34 and the type strain of Vibrio diazotrophicus. Originally A34 was thought to be a new species; however, recent DNA-DNA hybridization data have proven that A34 belongs to V. diazotrophicus. Class BC33 contained six strains, including the type strain of Vibrio campbellii.

Class BC35 accommodated Vibrio hispanicus and three other strains. BC38 merged the type strains of Vibrio orientalis and Vibrio natriegens. Originally the type strains of $V$. orientalis and Vibrio nigripulchritudo were grouped together with another five strains of uncertain taxonomic position (Thompson et al., 2001). BC39 grouped V. fischeri and Photobacterium iliopiscarius and so did the previous clustering (Thompson et al., 2001). BC40 consisted of four Vibrio pomeroyi strains including the type strain, but two further strains of this species were found in BC42. This is interesting in that these two strains (LMG 21351 and LMG 21352) differ in fAFLP patterns and the DNA-DNA hybridization data, suggesting that they are in fact at the outskirts of the species $V$. pomeroyi (Thompson et al., 2003c). Classes BC43 to BC45 all suffered from the same problem, namely merging of more the one type strain. Oddly enough, Salinivibrio costicola and Vibrio xuii, which are such different species, were included in the same class, BC43. On the other hand, BC46 consisted of three Vibrio tapetis strains which were previously merged with Vibrio penaeicida and Vibrio rumoiensis in the so-called fAFLP cluster A58. In the present analysis $V$. rumoiensis appeared in BC54 and V. penaeicida in BC57. Classes BC48, BC49, BC50, BC52 and BC58 contained Vibrio nereis, Vibrio metschnikovii, Vibrio aestuarianus, Vibrio cholerae and Vibrio mimicus, respectively. All these species had previously formed clusters on their own (Thompson et al., 2001).

For the Vibrio/AFLP dataset, there was good overall agreement between the classification based on the minimization of SC and the classification as described by Thompson et al. (2001) based on Ward's hierarchical clustering algorithm.
However, it is quite clear that certain heterogeneous fAFLP groups, e.g. A12, A13, A56, A58 and 59, were totally repartitioned with the new classification. Obviously, many of the repartitioned strains had been given only tentative names. For instance, A59 contained the type strain of $V$. tubiashii and thus we assumed that all strains clustering together with this type strain at the level of as low as $45 \%$ similarity would belong to the species $V$. tubiashii. Apparently this assumption goes beyond the discrimination of fAFLP and the mathematical algorithms used for fAFLP pattern analysis. Additionally, in other cases (e.g. BC46, BC54 and BC57) the new classification has repartitioned into separate classes type strains which were grouped together in a former analysis (Thompson et al., 2001). Some 'hidden' relationships have been disclosed with the help of the BinClass classification. This is the case for the former fAFLP groups A68 and A69, which appear in class BC3 (E. norvegicus). Likewise clusters $\mathrm{A} 9$ and $\mathrm{A} 60$ are merged in BC8 ( $V$. fortis), clusters $\mathrm{A} 34$ and $\mathrm{A} 57$ in BC30 ( $V$. diazotrophicus), and also clusters $\mathrm{A} 14$ and $\mathrm{A} 37$ in $\mathrm{BC} 27$ ( $V$. campbellii). All these four classes have been validated by DNA-DNA hybridization data which showed that these classes include strains from the same species (Thompson et al., 2002, 2003a).

\section{Conclusion}

In this report we have demonstrated the applicability of sliding-window discretization in combination with the minimization of SC for the classification of genotypic fingerprint patterns. As such, this new classification strategy forms a complementary alternative to the hierarchical cluster algorithms that are commonly used in bacterial taxonomy. Comparison of the classifications for the same set of fAFLP fingerprint patterns by different classification strategies has revealed that there was a good overall correlation between the alternative groupings, but that no single classification managed to reflect all the taxonomic relationships within the Vibrionaceae.

\section{ACKNOWLEDGEMENTS}

F. L. Thompson worked on a PhD scholarship (no. 2008361/98-6) from the Conselho Nacional de Desenvolvimento Científico e Tecnológico $(\mathrm{CNPq})$, Brazil. J. Swings acknowledges grants from the Fund for Scientific Research (FWO), Belgium. The research of T. Koski has been supported by the Swedish Research Council (VR/ NT). The research of M. Gyllenberg was supported by the Academy of Finland.

\section{REFERENCES}

Anderberg, M. R. (1973). Cluster Analysis for Applications. New York: Academic Press.

Arias, C. R., Verdonck, L., Swings, J., Garay, E. \& Aznar, R. (1997). Intraspecific differentiation of Vibrio vulnificus biotypes by amplified fragment length polymorphism and ribotyping. Appl Environ Microbiol 63, 2600-2606. 
Austin, B., Dawyndt, P., Gyllenberg, M., Koski, T., Lund, T., Swings, J. \& Thompson, F. L. (2004). Sliding window discretization: a new method for multiple band matching of bacterial genotyping fingerprints. Bull Math Biol 66, 1575-1596.

Ben-Haim, Y., Thompson, F. L., Thompson, C. C., Cnockaert, M. C., Hoste, B., Swings, J. \& Rosenberg, E. (2003). Vibrio coralliilyticus sp. nov., a temperature-dependent pathogen of the coral Pocillopora damicornis. Int J Syst Evol Microbiol 53, 309-315.

Buchanan, R. E. (1925). General Systematic Bacteriology. Baltimore: Williams \& Wilkins.

Cover, T. M. \& Thomas, J. A. (1991). Elements of Information Theory. New York: Wiley.

Dice, L. R. (1945). Measures of the amount of ecological association between species. Ecology 26, 297-302.

Gomez-Gil, B., Thompson, F. L., Thompson, C. C. \& Swings, J. (2003). Vibrio pacinii sp. nov., from cultured aquatic organisms. Int J Syst Evol Microbiol 53, 1569-1573.

Gyllenberg, M., Koski, T. \& Verlaan, M. (1997a). Classification of binary vectors by stochastic complexity. J Multivariate Anal 63, $47-72$.

Gyllenberg, H. G., Gyllenberg, M., Koski, T., Lund, T., Schindler, J. \& Verlaan, M. (1997b). Classification of Enterobacteriaceae by minimization of stochastic complexity. Microbiology 143, 721-732.

Gyllenberg, H. G., Gyllenberg, M., Koski, T. \& Lund, T. (1998). Stochastic complexity as a taxonomic tool. Comput Methods Programs Biomed 56, 11-22.

Gyllenberg, H. G., Gyllenberg, M., Koski, T., Lund, T. \& Schindler, J. (1999). Enterobacteriaceae taxonomy approached by minimization of stochastic complexity. Quantitative Microbiol 1, 157-170.

Gyllenberg, M., Koski, T. \& Lund, T. (2001). BinClass: a software package for classifying binary vectors. User's guide. TUCS Technical Report 411 http://www.tucs.fi/publications/techreports/TR411.php.

Gyllenberg, M., Dawyndt, P., Koski, T., Lund, T., Thompson, F., Austin, B. \& Swings, J. (2002). New methods for the analysis of binarized BIOLOG GN data of Vibrio species: minimization of stochastic complexity and cumulative classification. Syst Appl Microbiol 25, 403-415.

Janssen, P., Coopman, R., Huys, G., Swings, J., Bleeker, M., Vos, P., Zabeau, M. \& Kersters, K. (1996). Evaluation of the DNA fingerprinting method AFLP as a new tool in bacterial taxonomy. Microbiology 142, 1881-1893.

Liston, J., Wiebe, W. J. \& Colwell, R. R. (1963). Quantitative approach to the study of bacterial species. J Bacteriol 85, 1061-1070.

Mácian, M. C., Garay, E., Gonzalez-Candelas, F., Pujalte, M. J. \& Aznar, R. (2000). Ribotyping of Vibrio populations associated with cultured oysters (Ostrea edulis). Syst Appl Microbiol 23, 409-417.

Priest, F. \& Austin, B. (1993). Modern Bacterial Taxonomy, 2nd edn. London: Chapman \& Hall.
Sawabe, T., Thompson, F. L., Heyrman, J. \& 7 other authors (2002). Fluorescent amplified fragment length polymorphism and repetitive extragenic palindrome-PCR fingerprinting reveal host-specific genetic diversity of Vibrio halioticoli-like strains isolated from the gut of japanese Abalone. Appl Environ Microbiol 68, 4140-4144.

Sawabe, T., Hayashi, K., Moriwaki, J., Thompson, F. L., Swings, J. \& Christen, R. (2004). Vibrio neonatus sp. nov. and Vibrio ezurae sp. nov. isolated from the gut of Japanese abalones. Syst Appl Microbiol 27, 527-534.

Schiewe, M. H., Trust, T. J. \& Crosa, J. H. (1981). Vibrio ordalii sp. nov.: a causative agent of vibriosis in fish. Curr Microbiol 6 , 343-348.

Sneath, P. H. A. (1984). Bacterial Nomenclature. In Bergey's Manual of Systematic Bacteriology, vol. 1, pp. 19-23. Edited by N. R. Krieg \& J. G. Holt. Baltimore: Williams \& Wilkins.

Sneath, P. H. A. \& Sokal, R. R. (1973). Numerical Taxonomy: the Principles and Practice of Numerical Classification. San Francisco: W. H. Freeman.

Thompson, F. L., Hoste, B., Vandemeulebroecke, K. \& Swings, J. (2001). Genomic diversity amongst Vibrio isolates from different sources determined by fluorescent amplified fragment length polymorphism. Syst Appl Microbiol 24, 520-538.

Thompson, F. L., Hoste, B., Thompson, C. C., Goris, J., GomezGil, B., Huys, L. \& Swings, J. (2002). Enterovibrio norvegicus gen. nov., sp. nov., isolated from the gut of turbot (Scophthalmus maximus) larvae: a new member of the family Vibrionaceae. Int J Syst Evol Microbiol 52, 2015-2022.

Thompson, F. L., Thompson, C. C., Hoste, B., Vandemeulebroecke, K., Gullian, M. \& Swings, J. (2003a). Vibrio fortis sp. nov. and Vibrio hepatarius sp. nov., isolated from aquatic animals and the marine environment. Int J Syst Evol Microbiol 53, 1495-1501.

Thompson, F. L., Thompson, C. C. \& Swings, J. (2003b). Vibrio tasmaniensis sp. nov., isolated from atlantic salmon (Salmo salar L.). Syst Appl Microbiol 26, 65-69.

Thompson, F. L., Thompson, C. C., Li, Y., Gomez-Gil, B., Vandenberghe, J. \& Swings, J. (2003c). Vibrio kanaloae sp. nov, Vibrio pomeroyi sp. nov. and Vibrio chagasii sp. nov., from sea water and marine animals. Int J Syst Evol Microbiol 53, 753-759.

Thompson, F. L., Li, Y., Gomez-Gil, B. \& 8 other authors (2003d). Vibrio neptunius sp. nov., Vibrio brasiliensis sp. nov. and Vibrio xuii sp. nov., isolated from the marine aquaculture environment (bivalves, fish, rotifers and shrimps). Int J Syst Evol Microbiol 53, 245-252.

Vandenberghe, J., Verdonck, L., Robles-Arozarena, R. \& 7 other authors (1999). Vibrios associated with Litopenaeus vannamei larvae, postlarvae, broodstock and hatchery probionts. Appl Environ Microbiol 65, 2592-2597.

Ward, J. H. (1963). Hierarchical grouping to optimize an objective function. J Am Stat Assoc 58, 236-244. 